\title{
Terminal phalangeal osteosclerosis and rheumatoid arthritis
}

\author{
Michael Currat MD, Bérengère Aubry-Rozier MD, Jean Dudler MD
}

Competing interests: None declared.

This article has been peer reviewed.

Affiliations: From the Departments of Internal Medicine (Currat) and Rheumatology (AubryRozier, Dudler), Centre hospitalier universitaire vaudois, Lausanne,

Switzerland

Correspondence to: Dr. Jean Dudler, jean.dudler@chuv.ch

CMAJ 2011. DOI:10.1503 /cmaj.091189

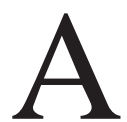

58-year-old woman was admitted to our rheumatology ward for evaluation and treatment of seronegative polyarthritis. She had a three-year history of peripheral symmetric arthritis involving her wrists, metacarpophalangeal and proximal interphalangeal joints, knees and ankles. She had previously undergone unsuccessful trials of corticosteroids, methotrexate, leflunomide and two antitumour necrosis factor agents. On admission, she complained of fatigue, along with severe bilateral joint pain and swelling of her hands, elbows and knees. She also had symptoms of severe Raynaud syndrome affecting her hands.

Examination showed symmetric synovitis of her wrists, elbows and knees, as well as most metacarpophalangeal and proximal interphalangeal joints, but was otherwise normal. Blood tests showed normochromic normocytic anemia with elevated inflammatory parameters (erythrocyte sedimentation rate and C-reactive protein level). Rheumatoid factors, and anti-cyclic citrullinated peptide and antinuclear antibodies were negative. Hand radiographs (Figure 1) showed striking sclerosis of the terminal phalanges; this sclerosis was not seen in other surveyed bones including those of the elbows, pelvis, knees and feet, nor was there evidence of calcinosis. This finding was diagnosed as terminal phalangeal osteosclerosis, a normal variant. The patient also had classic seronegative rheumatoid arthritis. The radiographic appearance of the osteosclerosis was probably enhanced by severe osteopenia related to the patient's rheumatoid arthritis and previous corticotherapy.

Terminal phalangeal osteosclerosis is a localized and sporadic form of endosteal bone formation occurring typically in the distal phalanges ${ }^{1}$ and is not pathognomonic of any disease. Its specific location differentiates it from generalized endosteal new-bone formation (e.g., in adult sickle cell disease). A similar radiographic appearance may be found with superimposed calcinosis over the tuft, as seen occasionally in connective tissue disease.

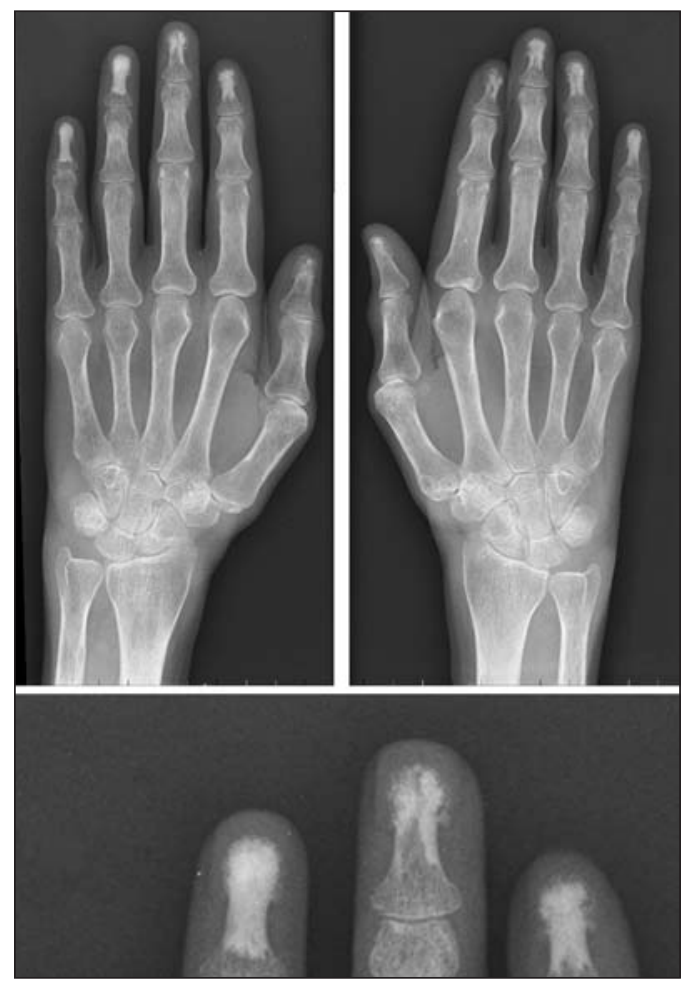

Figure 1: Radiographs of the hands of a 58-year-old woman with seronegative rheumatoid arthritis showing osteosclerosis of the terminal phalanges.

Although terminal phalangeal osteosclerosis was initially believed to be related to and more prevalent in collagen vascular diseases, ${ }^{2}$ later reports failed to support this association. ${ }^{3,4}$ Terminal phalangeal osteosclerosis should be regarded as a normal and common variant, ${ }^{1}$ with more than $50 \%$ of the population over 44 years of age showing some evidence of this condition. ${ }^{3}$

\section{References}

1. Forrester DM, Brown JC. The radiology of joint disease. 3rd ed. Philadelphia (PA): W.B. Saunders Company; 1987. p. 78.

2. Goodman N. The significance of terminal phalangeal osteosclerosis. Radiology 1967;89:709-12.

3. Halim W, Van Der Korst JK, Valkenburg HA, et al. Terminal phalangeal osteosclerosis. Ann Rheum Dis 1975;34:82-6.

4. Jacob J, Sartoris D, Kursunoglu S, et al. Distal interphalangeal joint involvement in rheumatoid arthritis. Arthritis Rheum 1986;29:10-5. 